\section{Public Health} Genomics
Public Health Genomics 2014;17:48-60

DOI: 10.1159/000356567
Received: April 11, 2013

Accepted after revision: October 21, 2013

Published online: January 14, 2014

\title{
Interest and Informational Preferences Regarding Genomic Testing for Modest Increases in Colorectal Cancer Risk
}

\author{
A.E. Anderson ${ }^{a} \quad$ K.G. Flores ${ }^{f} \quad$ W. Boonyasiriwat ${ }^{a}{ }^{a} \quad$ A. Gammon ${ }^{a} \quad$ W. Kohlmann ${ }^{a}$ \\ W.C. Birmingham ${ }^{\text {e M.D. Schwartz }}{ }^{\text {h, } i} \quad$ J.Samadder ${ }^{a, b} \quad$ K. Boucher ${ }^{a, c}$ \\ A.Y. Kinney ${ }^{a, b, f, g}$ \\ ${ }^{a}$ Huntsman Cancer Institute, Departments of ${ }^{\mathrm{b}}$ Internal Medicine, ${ }^{\mathrm{c} O n c o l o g i c a l ~ S c i e n c e s, ~ U n i v e r s i t y ~ o f ~ U t a h, ~ a n d ~}$ \\ ${ }^{\mathrm{d}}$ Department of Internal Medicine, University of Utah School of Medicine, Salt Lake City, Utah, and ${ }^{\mathrm{e}}$ Department of \\ Psychology, Brigham Young University, Provo, Utah, ${ }^{f}$ University of New Mexico Cancer Center, and ${ }^{9}$ Department of \\ Internal Medicine, University of New Mexico, Albuquerque, N.Mex., ${ }^{\text {h}}$ Department of Oncology, Georgetown \\ University School of Medicine, and 'Lomardi Comprehensive Cancer Center, Washington D.C., USA; ${ }^{j}$ Faculty of \\ Psychology, Chulalongkorn University, Bangkok, Thailand
}

\section{Key Words}

Behavior change $\cdot$ Cancer risk - Colorectal cancer .

Direct-to-consumer · First-degree relatives · Genomic · SNP

\begin{abstract}
Background/Aims: This study explored the interest in genomic testing for modest changes in colorectal cancer risk and preferences for receiving genomic risk communications among individuals with intermediate disease risk due to a family history of colorectal cancer. Methods: Surveys were conducted on 272 men and women at intermediate risk for colorectal cancer enrolled in a randomized trial comparing a remote personalized risk communication intervention (TeleCARE) aimed at promoting colonoscopy to a generic print control condition. Guided by Leventhal's Common Sense Model of Self-Regulation, we examined demographic and psychosocial factors possibly associated with interest in SNP testing. Descriptive statistics and logistic regression models were used to identify factors associated with interest
\end{abstract}

in SNP testing and preferences for receiving genomic risk communications. Results: Three-fourths of participants expressed interest in SNP testing for colorectal cancer risk. Testing interest did not markedly change across behavior modifier scenarios. Participants preferred to receive genomic risk communications from a variety of sources: printed materials (69.5\%), oncologists (54.8\%), primary-care physicians (58.4\%), and the web (58.1\%). Overall, persons who were unmarried $(p=0.029)$, younger $(p=0.003)$ and with greater cancer-related fear ( $p=0.019$ ) were more likely to express interest in predictive genomic testing for colorectal cancer risk. In a stratified analysis, cancer-related fear was associated with the interest in predictive genomic testing in the intervention group $(p=0.017)$, but not the control group. Conclusions: Individuals with intermediate familial risk for colorectal cancer are highly interested in genomic testing for modest increases in disease risk, specifically unmarried persons, younger age groups and those with greater cancer fear.

(c) 2014 S. Karger AG, Basel

\section{KARGER}

E-Mail karger@karger.com www.karger.com/phg
(C) 2014 S. Karger AG, Basel

$1662-4246 / 14 / 0171-0048 \$ 39.50 / 0$
Anita Y. Kinney, $\mathrm{PhD}$

Department of Internal Medicine, University of New Mexico Cancer Center 1 University of New Mexico MSC07 4025

Albuquerque, NM 87108 (USA)

E-Mail AYKinney@ salud.unm.edu 


\section{Introduction}

Hereditary risk plays a role in approximately $30 \%$ of all colorectal cancer cases [1]. To date, genetic testing for hereditary colon cancer risk has focused on the identification of families with high-risk hereditary colon cancer syndromes, such as Lynch syndrome and familial adenomatous polyposis. The lifetime colon cancer risks associated with these conditions are significant if there is no intervention (up to $80 \%$ with Lynch syndrome and $100 \%$ with classic familial adenomatous polyposis) [1, 2]. Guidelines for ordering genetic testing to assist in the identification of these high-risk families, as well as appropriate screening and risk-reduction strategies for affected individuals, are available from the National Comprehensive Cancer Network, consensus statements and expert review papers in the literature [1-3].

However, as only $2-5 \%$ of colorectal cancer cases are accounted for by high-risk hereditary conditions, much of the heritability of colorectal cancer remains unexplained $[1,4]$. Family history impacts the colorectal cancer risk in relatives outside of high-risk syndromes depending on the number, degree and age at diagnosis of affected relatives $[4,5]$. While having one or more firstdegree relatives with colorectal cancer is known to increase the risk, recent research shows that combinations of one affected first-degree relative plus multiple secondor third-degree relatives with colorectal cancer can also increase colorectal cancer risk by $2-5$ fold [5]. Currently, available screening guidelines for individuals at moderately increased risk for colorectal cancer, referred to here as intermediate risk, are based on family history $[2,6]$. However, some individuals may be unaware of their family history of colon cancer and, thus, their intermediate cancer risk. Identifying the genetic causes of moderate increases in familial colorectal cancer risk may allow more individuals to be aware of their risk through genetic testing, rather than relying solely on family history analysis, and facilitate further tailoring of screening and risk-reduction recommendations.

Research surrounding the genetic factors conferring a moderately increased risk for colorectal cancer has focused on SNPs. SNPs, the most common type of genetic variation in the human genome, contribute to the multifactorial etiology of chronic diseases, such as gene-environment interactions [7]. Genome-wide association studies have identified SNPs which occur more or less frequently in disease versus control populations, and the odds ratios (ORs) from these studies are being used to create risk estimates. Generally, SNP testing identifies

Interest in Genomic Testing for Colorectal Cancer Risk small increases in disease risk for a large number of people. Capitalizing on these recent genomic discoveries, some for-profit companies now offer direct-to-consumer (DTC) SNP-based testing for common health-related conditions and traits, including colorectal cancer. Such companies encourage consumers to manage personal health risks and make informed health decisions by learning their SNP results.

Controversy surrounds the clinical validity (degree to which a test accurately predicts disease risk) and utility (whether test findings benefit disease diagnosis, treatment and management) of SNP testing. Opponents argue that testing for SNPs is of little clinical value because known genetic variations only account for a small portion of the heritability of common diseases as well as the fact that there are significant environmental contributions to such diseases [8]. Proponents, however, claim genetic testing for even modest changes in disease risk may motivate health behavior change beyond typical prevention efforts [9]. Regardless of limited evidence demonstrating the benefits of this type of genomic testing, companies continue to market DTC predictive SNP testing promising health benefits for consumers. A 2011 report from the Genetics and Public Policy Center listed 8 companies providing DTC testing specifically for colorectal cancer and 2 companies providing DTC testing for colorectal cancer only through a physician. Of the 8 DTC companies, 2 companies offered genetic counseling services without additional cost, while 3 companies offered genetic counseling services with additional cost [10]. Only 3 of the DTC-testing websites identified the SNPs associated with colorectal cancer risk used in testing; the number of SNPs tested ranged from 4-8 [11, 12]. The risks of colorectal cancer associated with these SNPs are relatively small and differ by racial/ethnic group [11]. Actual public interest in DTC genetic testing services is highly variable with market size estimates ranging from USD 20-730 million [13, 14]. Basic DTC genomic testing is relatively affordable with personal genome sequencing available for only USD 99 [11]. The current market fluctuates with new companies emerging, companies dismantling or companies changing their health-related focus within a relatively short time frame [15]. Understanding the public's interest in and attitudes toward SNP testing is crucial for the effective and appropriate translation of genomic testing to clinical practice and policy, especially in light of the rapid expansion of DTC testing [13].

Evidence about consumer interest in and attitudes toward genomic testing is conflicting. Generally, consum- 
ers are interested in tests that cost less and indicate higher increases in disease risk rather than a decrease in risk $[16,17]$. Individuals who have a regular physician, higher perceived benefits of genetic testing and higher levels of cancer worry show more interest in SNP testing [16]. Perceived advantages of genetic testing from various study populations include: motivation for adopting a healthier lifestyle, sharing genetic risk information with family members and assisting healthcare providers in monitoring their patients' health $[18,19]$. A recent qualitative study found that consumers prefer healthcare professionals to provide genomic risk communications rather than print or computer materials [17]. Such insights can direct effective integration of SNP testing into clinical practice for potential behavior change and public health impacts.

Although the clinical validity and utility of genomic testing for known high-risk hereditary syndromes (e.g. familial adenomatous polyposis and Lynch syndrome) are well established [3], most familial colorectal cancer families have no identifiable genetic etiology. Predictive testing of SNPs associated with small to moderate increases in colorectal cancer risk is commercially available, but it is unknown how these tests motivate colorectal cancer screening and other healthy lifestyle behaviors. As an initial step in the translational pathway, it is important to understand factors associated with consumer interest in colorectal cancer susceptibility SNP testing and consumers' preferences for receiving genomic risk communications. Our study was guided by the Common Sense Model of Self-Regulation of Health and Illness [20]. This model underscores the central roles of cognitions and emotions in health-related decisions and actions, such as the complex information processing associated with genomic testing decisions. Genomic test results, specifically SNP testing, may largely influence an individual's threat representation about colorectal cancer, beyond family history alone [21]. Little is known about the characteristics of those who seek SNP testing for colorectal cancer susceptibility and their reasons for seeking testing. In the current analysis, we examined interest in genomic testing for colorectal cancer risk among relatives of colorectal cancer patients (i.e. those at intermediate risk) and factors associated with interest in testing. We also assessed preferences for receiving genomic risk communications seeing as genomic testing is associated with substantial information demands. Based on the Common Sense Model of Self-Regulation of Health and Illness, we hypothesized that in addition to sociodemographic factors, both cognitions and emotions would be associated with interest in SNP testing for colorectal cancer risk.

\section{Methods}

\section{Study Sample}

Our study sample was drawn from male and female participants enrolled in the Family Colorectal Cancer Risk Awareness and Risk Education Project (Family CARE). The Family CARE project is an on-going randomized, controlled trial investigating whether a personalized, remote, theoretically-based risk assessment and telephonic counseling intervention (TeleCARE) is more effective at motivating colonoscopy screening than a mailed, low-intensity targeted print message delivered to individuals who are considered at intermediate familial risk for colorectal cancer. The intensive colorectal cancer risk assessment intervention included a 30-45 min telephone counseling session with a certified genetic counselor, a 4-page tailored visual aid to use during the counseling session and a tailored follow-up letter. The minimal intervention (control) consisted of a targeted educational brochure defining colorectal cancer and discussing familial cancer risk and the role of colonoscopy in preventing colorectal cancer. More detailed descriptions of the study recruitment, intervention, theoretical and practical rationale, and methods is documented elsewhere [22, 23]. Eligible Family CARE study participants were recruited from 5 state cancer registries - Utah, Idaho, Colorado, New Mexico, and California - the Huntsman Cancer Center and Intermountain Health Care systems in Utah. Recruitment occurred in 3 stages by first contacting colorectal cancer patients in the state cancer registries, then requesting contact information for patients' family members and finally contacting family members directly. Briefly, eligibility criteria included: (1) age 30-74; (2) having either one first-degree relative diagnosed with colorectal cancer before age 60 , or one first-degree relative and an additional second-degree relative diagnosed at any age; (3) no prior cancer diagnosis other than non-melanoma skin cancer; (4) no colonoscopy in the past 5 years; (5) awareness of colorectal cancer family history; (6) mental competence, and (7) ability to read and speak English fluently. All participants were overdue for a screening colonoscopy based on their family history of the disease. Families with known familial adenomatous polyposis or meeting Amsterdam criteria for Lynch syndrome were excluded from the study. Participants considered in the current analysis included only those who completed the 9-month follow-up questionnaire and answered a series of questions on SNP testing. Nearly all of the participants were non-Hispanic white; therefore, the study was restricted to this group (for whom the findings are generalizable), resulting in a final sample size of 272 .

\section{Independent Variables}

The Common Sense Model of Self-Regulation contends that a health threat (in this case, familial colorectal cancer risk) generates 2 parallel processes - the cognitive process and the emotional process $[20,21]$. Based on these parallel processes, we selected the cognitive processes of perceived control, perceived severity, perceived susceptibility, and the emotional processes of cancer worry, fear, optimism, and anxiety as probable factors associated with interest in predictive SNP testing. We report Cronbach's alphas as measures of the multi-item scales' internal reliability for our study population below.

\section{Cognitive Factors}

Perceived Control. Perceived behavioral control was assessed with a single-item measure: 'How much control do you think you
Anderson et al. 
have over whether you can prevent colorectal cancer?' Response options were 'no control', 'some control' or 'complete control' [24].

Perceived Severity. Perceptions about the severity of colorectal cancer were measured using the following 4 items adapted from the Risk Behavior Diagnosis scale [25]: 'I believe that colorectal cancer is serious', 'I believe that colorectal cancer is harmful', 'I believe that colorectal cancer is a significant disease', and 'I believe that colorectal cancer has serious negative consequences' (Cronbach's alpha $=0.94)$. Response options were on a 5 -point Likert scale ranging from 'strongly disagree' to 'strongly agree'.

Perceived Susceptibility. Perceived susceptibility was measured using the following 4 items adapted from the Risk Behavior Diagnosis scale [25]: 'I am at risk for getting colorectal cancer', 'It is possible that I will get colorectal cancer', 'I am susceptible to getting colorectal cancer, and 'It is likely I will get colorectal cancer' (Cronbach's alpha $=0.83$ ). Response options were on a 5-point Likert scale ranging from 'strongly disagree' to 'strongly agree'.

\section{Emotional Factors}

Trait Anxiety. Trait anxiety was assessed with an 8-item subscale taken from the NEO Personality Inventory [26]. The measure demonstrated acceptable internal consistency (Cronbach's alpha $=0.79)$ in the present sample.

Cancer Worry. We assessed frequency and intensity of cancer worry with a 3-item measure [27]. Worry frequency was measured using a single-item that asked participants how often they worried about developing colorectal cancer in their lifetime. Response options were on a 5-point Likert scale ranging from 'never' to 'all of the time'. Two-items assessed worry intensity by asking participants: 'How bothered are you by thinking about getting colorectal cancer' and 'How worried are you about getting colorectal cancer?' Response options were on a 5-point Likert scale ranging from 'not at all' to 'extremely'. Worry frequency and worry intensity were combined to create a composite worry variable (Cronbach's alpha $=0.80$ ).

Fear. We measured participants' fear about developing colorectal cancer using the unpublished 6-item Negative Affect in Risk scale developed by Hay et al. [28] at Memorial Sloan Kettering Cancer Center in New York City. The scale included the following statements: 'I get frightened when I think I could get colorectal cancer', 'Thinking about getting colorectal cancer makes me afraid', 'I get a bad feeling just thinking about the possibility of getting colorectal cancer', 'Thinking about my chances of getting colorectal cancer makes me uncomfortable', 'I dread getting colorectal cancer', and 'I can't think about getting colorectal cancer without feeling afraid'. Response options were on a 4-point Likert scale ranging from 'strongly disagree' to 'strongly agree'. The scale demonstrated high internal consistency (Cronbach's alpha $=0.93$ ) in our sample.

Optimism. Optimism was measured using the following 6 items adapted from the Life Orientation Test-Revised [29]: 'In uncertain times, I usually expect the best'; 'If something can go wrong for me, it will'; 'I'm always optimistic about the future'; 'I hardly ever expect things to go my way'; 'I rarely count on good things happening to me'; and 'Overall, I expect more good things to happen to me than bad' (Cronbach's alpha $=0.87$ ). Response options were on a 5-point Likert scale ranging from 'strongly disagree' to 'strongly agree'.

Interest in Genomic Testing for

Colorectal Cancer Risk
Additional Variables

Sociodemographics. We assessed age, gender, marital status, income, education, health insurance status, rural/urban residence, and access to a personal healthcare provider. Rural/urban residence was based on Rural-Urban Commuting Area Codes at the census tract level [30]. Race/ethnicity was not included in the analysis because almost all participants were non-Hispanic white, thus, limiting statistical power to draw meaningful conclusions regarding racial/ethnic differences in genomic testing interest. Finally, we included whether participants were in the intervention or control arm of the Family CARE study. The study arm received a multifaceted genomic risk and behavior change intervention over the telephone with a certified genetic counselor (TeleCARE) and mailed tailored materials while the control arm only received a brochure on familial colorectal cancer risk and colonoscopy screening.

Risk Behavior Profile. Three modifiable risk factors - physical activity, BMI and smoking status - were used to create a studyspecific risk behavior index. Participants were considered compliant with the 2008 Physical Activity Guidelines if they reported at least $150 \mathrm{~min}$ a week of moderate-intensity exercise, or $75 \mathrm{~min}$ a week of vigorous-intensity aerobic physical activity, or an equivalent combination of moderate and vigorous intensity aerobic activity [31]. Those meeting these guidelines received a 1 and participants not meeting these guidelines received a 0 . We used the standard BMI weight categories as suggested by the Centers for Disease Control and Prevention [32]. Those in the underweight and average weight categories received a 1 while participants in the overweight and obese categories received a 0 . Participants not currently smoking received a 1 and current smokers received a 0 . Scores ranged from 0 (all risk behaviors) and 3 (no risk behaviors). Similar risk behavior indices were utilized in previous studies [33, 34].

\section{Outcome Variables}

Interest in Genomic Testing. Prior to responding to a series of questions on interest in SNP testing and preferences for receiving genomic risk communications, participants were briefly introduced to SNPs and genetic testing. The information covered how genes influence health, genetic testing and disease risk, SNPs, genetic testing processes, and the predictive power of SNPs for colorectal cancer risk. To determine overall interest in genomic testing for modest changes in colorectal cancer risk, we first asked participants 'If SNP testing could tell you that you may have a slightly increased risk of developing colorectal cancer, how likely is it that you would want a SNP test?' with 4 response options - 'I would definitely not have the test,' 'I would probably not have the test,' 'I would probably have the test,' and 'I would definitely have the test.' Due to small numbers in the first level, responses were dichotomized to those not interested in SNP testing and those interested in SNP testing.

We also examined if interest in SNP testing varied according to the potential for colorectal cancer risk reduction through behavior change - regular colorectal cancer screenings, taking medications, and diet or exercise. Finally, we assessed participants' preferences regarding modes of genomic risk communications including print or written sources, web-based sources, computer kiosk touch screen, in person with a nurse, in person with a primary care physician, in person with an oncologist and in person with a genetic counselor. The survey briefly explained the function of genetic counselors. 
Table 1. Characteristics of Family CARE participants by completion status of the 9-month follow-up survey that assessed interest in predictive SNP testing for colorectal cancer risk

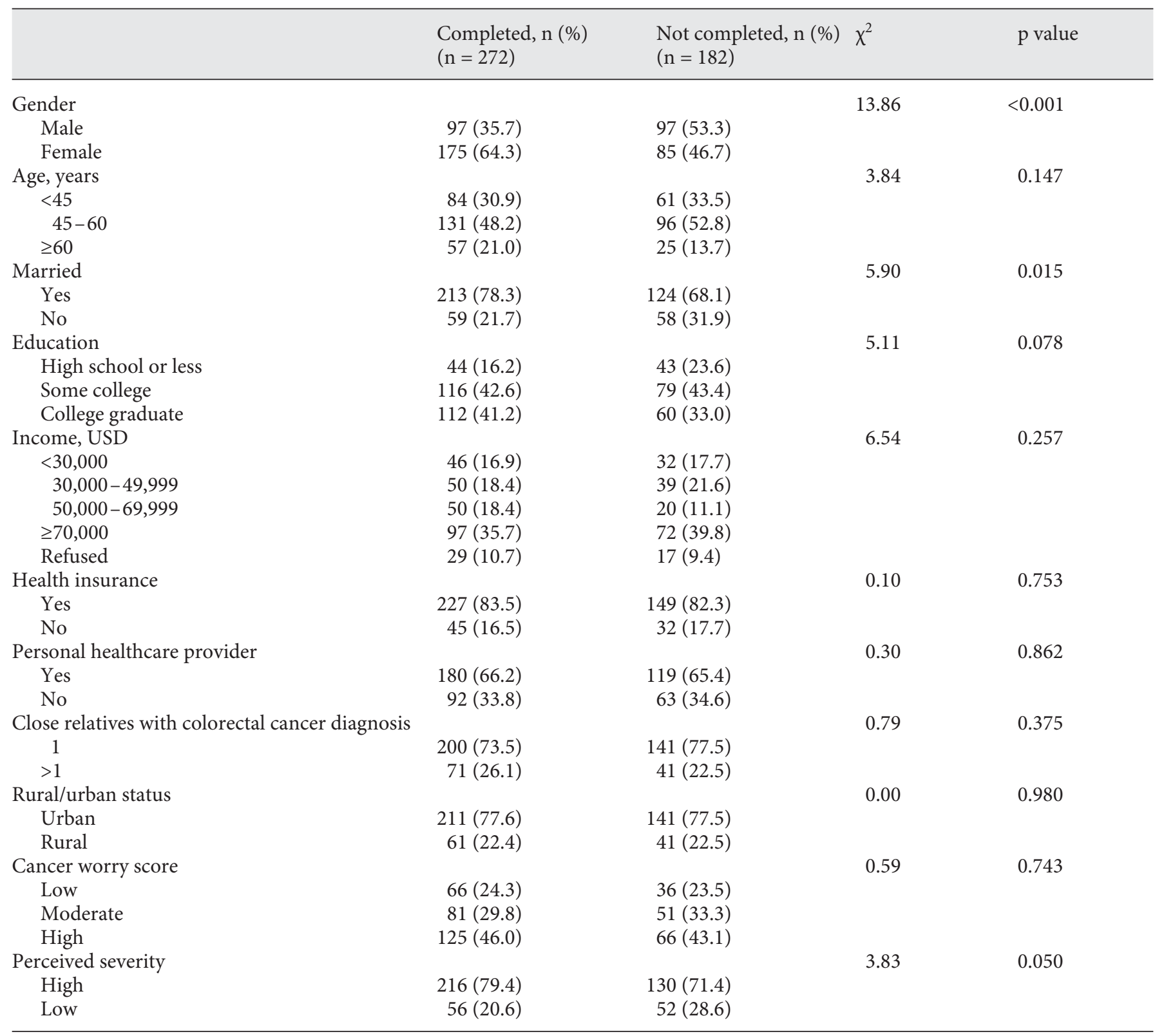

\section{Statistical Analysis}

Descriptive statistics were used to characterize sociodemographic, clinical and psychological variables of the study population, delineate interest in SNP testing generally and according to behavioral modifiers specifically as well as preferences for receiving genomic risk communications. Differences in the characteristics between survey respondents and nonrespondents were assessed using $\chi^{2}$ tests. Unadjusted ORs and 95\% confidence intervals (CIs) were used to identify each variable's association with interest in SNP testing in the overall study population and by intervention subgroup. Multivariable logistic regression models were used to identify factors that were independently associated with interest in SNP testing. Variables with crude statistical significance $(\mathrm{p}<0.20)$ were entered into the regression model and backward elimination procedures were used to determine which variables remained in the model $(\mathrm{p}<0.10)$ [27]. We used generalized linear mixed models (Proc GLIMMIX) to account for familial clustering in the study design, but no design effect was observed. Further, in a sensitivity analysis using logistic regression models (Proc Logistic) without familial clustering, the OR estimates were within 0.01 points of one another. However, all study results presented here were calculated using generalized linear 


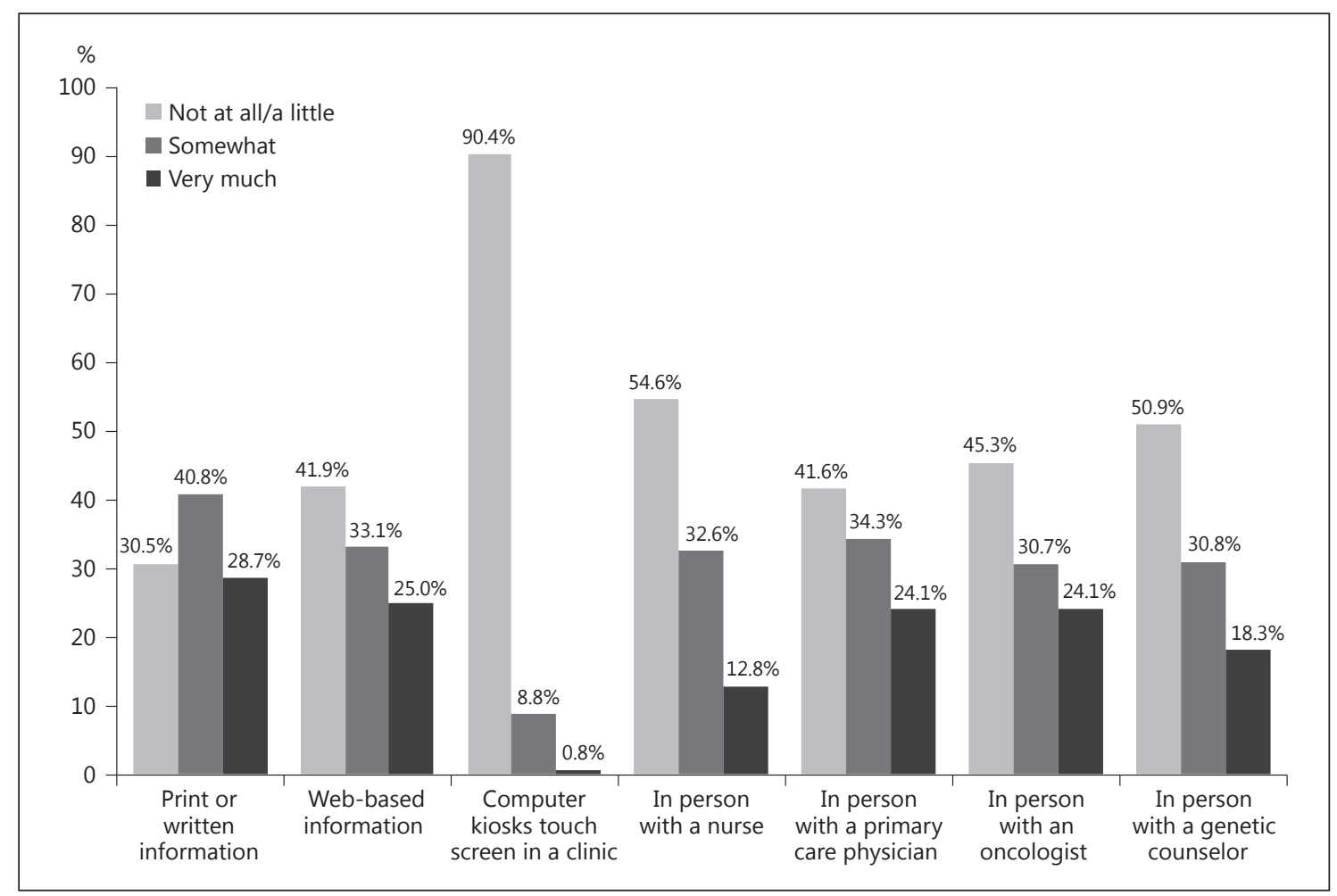

Fig. 1. Preferences for receiving genomic risk communication.

mixed models. Independent variables with a non-normal distribution were dichotomized or grouped into tertiles accordingly. Only participants with valid scale scores, as defined by validation studies for each measure, were included in the analyses. All analyses were performed using SAS 9.2 (SAS Institute Inc., Cary, N.C., USA, 2001).

\section{Results}

Participants that completed the 9-month assessment differed from participants that did not complete the 9-month survey by gender $\chi^{2}[(1, \mathrm{n}=454)=13.86, \mathrm{p}<$ $0.01]$ and marital status $\left.\chi^{2}[(1, \mathrm{n}=454)=5.90, \mathrm{p}=0.015)\right]$ (table 1). We found no significant differences in age group, education level, insurance status, rural/urban residence, number of relatives with colorectal cancer, cancer fear, cancer worry, and perceived susceptibility between those with completed 9-month assessments and those without.

Characteristics of the study population are presented in table 2 . In the overall study population, most participants were married (78\%), attended at least some college
(84\%), had health insurance coverage (83\%), reported access to a personal healthcare provider (66\%), lived in urban areas $(78 \%)$, and all had at least one first-degree relative with colorectal cancer. Approximately $64 \%$ of participants were female and, on average, participants were 51.2 years of age $(\mathrm{SD}=9.63$ years; range $34-74$ years). Overall, $74 \%$ of participants indicated at least some interest in predictive SNP testing for colorectal cancer. Specifically, $4 \%$ of participants said they would definitely not have SNP testing, 22\% said they would probably not have testing, $47 \%$ said they would probably have testing, and $27 \%$ said they would definitely have testing. Interest in SNP testing did not markedly change between behavior modification scenarios. More participants (77\%) expressed interest in genomic testing that would indicate whether or not their risk of colorectal cancer could be lowered by diet and exercise than having regular colonoscopies or taking medication (data not shown). The most commonly preferred sources of genomic risk information were print sources $(69.5 \%)$, oncologists (54.8\%), primary-care physicians $(58.4 \%)$, and web-based sources (58.1\%) (fig. 1). 


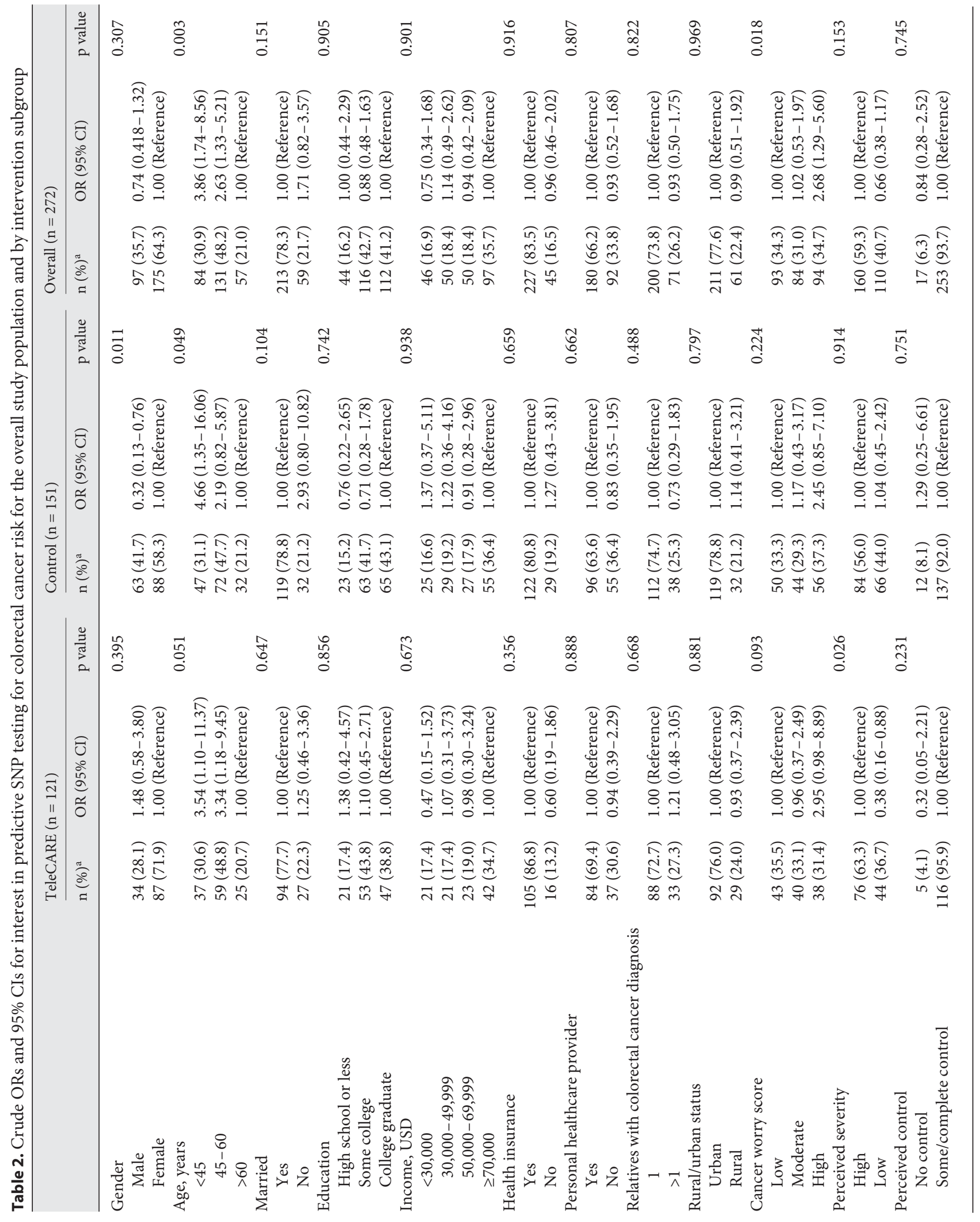




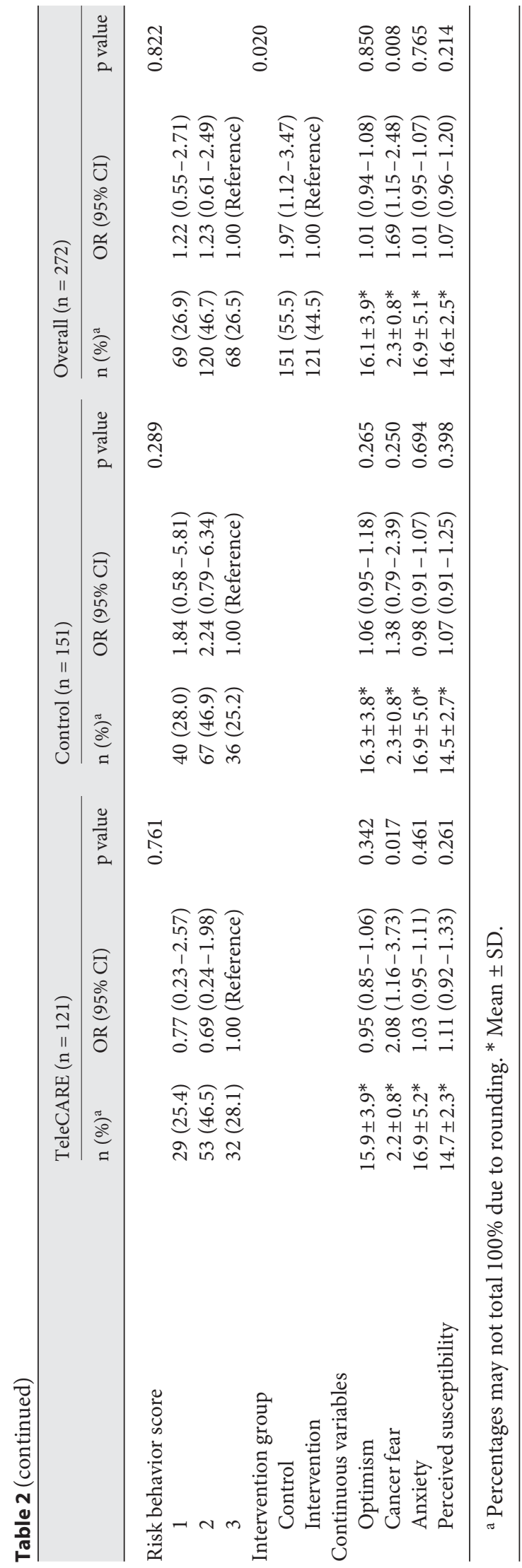

Interest in Genomic Testing for Colorectal Cancer Risk
Table 2 presents unadjusted ORs, $95 \%$ CIs and p values for each independent variable in the overall study population and by intervention subgroup. Age, marital status, worry, perceived severity, cancer fear, and intervention group met the criteria $(p<0.20)$ for entry into the logistic model for the overall study population. For the TeleCARE group, age, worry, perceived severity, and cancer fear were used in the logistic model. For the control group, gender, age and marital status were included in the model.

The final adjusted logistic models included variables $(\mathrm{p}<0.10)$ shown in table 3. Overall, unmarried individuals expressed greater interest in SNP testing than married participants $(\mathrm{OR}=2.49,95 \% \mathrm{CI}=1.10-5.61, \mathrm{p}=0.029)$. Participants less than 45 years indicated more interest in testing than those over 65 years $(\mathrm{OR}=4.26,95 \% \mathrm{CI}=$ $1.81-10.01, \mathrm{p}=0.003)$. Those in the control group were more interested in genomic testing than those in the intervention group $(\mathrm{OR}=2.05,95 \% \mathrm{CI}=1.12-3.74, \mathrm{p}=$ 0.021). Participants reporting higher levels of cancer fear were more likely to indicate interest in SNP testing than those with lower fear levels (OR $=1.61,95 \%$ CI $=1.09$ $2.43, \mathrm{p}=0.019)$. In a stratified analysis, the models differed by intervention subgroups. For the control group, age and marital status were associated with interest in SNP testing and males were less likely to express interest in SNP testing compared to females (OR $=0.30,95 \%$ $\mathrm{CI}=0.12-0.74, \mathrm{p}=0.011$ ). Only cancer fear was significantly associated with interest in SNP testing for the intervention group $(\mathrm{OR}=2.08,95 \% \mathrm{CI}=1.16-3.73, \mathrm{p}=$ $0.017)$. To assess the combined effect of risk perceptions and select emotions, we tested for interactions between perceived risk and worry as well as perceived risk and fear. However, we found no significant interactions.

\section{Discussion}

To our knowledge, this is the first study to measure interest in predictive SNP testing for modest changes in colorectal cancer risk and preferences for receiving genomic risk information among at-risk relatives of colorectal cancer patients. Overall interest in SNP testing was high in our sample, with approximately three-fourths of the study population expressing at least some interest in testing, despite only small to modest increases in disease risk. Participants in the Family CARE project were made aware of their increased familial risk for colorectal cancer, perhaps causing greater interest in genetic testing than in the general population. However, a small study of average 
Table 3. Adjusted logistic regression models for the overall study population and by intervention subgroup for interest in predictive SNP testing for colorectal cancer risk

\begin{tabular}{|c|c|c|c|}
\hline & Adjusted OR* & $95 \% \mathrm{CI}$ & p value* \\
\hline \multicolumn{4}{|l|}{ Overall } \\
\hline Married & & & 0.029 \\
\hline Yes & 1.00 & Reference & \\
\hline No & 2.49 & $1.10-5.61$ & \\
\hline Age, years & & & 0.003 \\
\hline$<45$ & 4.26 & $1.81-10.01$ & \\
\hline $45-60$ & 2.83 & $1.35-5.92$ & \\
\hline$>60$ & 1.00 & Reference & \\
\hline Intervention group & & & 0.021 \\
\hline Control & 2.05 & $1.12-3.74$ & \\
\hline Intervention & 1.00 & Reference & \\
\hline Cancer fear & 1.61 & $1.09-2.43$ & 0.019 \\
\hline \multicolumn{4}{|l|}{ Control } \\
\hline Married & & & 0.048 \\
\hline Yes & 1.00 & Reference & \\
\hline No & 4.09 & $1.01-16.55$ & \\
\hline Age, years & & & 0.041 \\
\hline$<45$ & 5.54 & $1.49-20.65$ & \\
\hline $45-60$ & 2.25 & $0.78-6.53$ & \\
\hline$>60$ & 1.00 & Reference & \\
\hline Gender & & & 0.011 \\
\hline Male & 0.30 & $0.12-0.74$ & \\
\hline Female & 1.00 & Reference & \\
\hline \multicolumn{4}{|l|}{ Intervention } \\
\hline Cancer fear & 2.08 & $1.16-3.73$ & 0.017 \\
\hline
\end{tabular}

risk primary care patients also found that $75 \%$ of participants expressed interest in SNP testing for increased colorectal cancer risk [17].

Interest in SNP testing did not appreciably change across behavior modification scenarios, with interest remaining close to $75 \%$. These findings are noteworthy in light of the recent debate surrounding genomic testing and the potential for health behavior change [35]. Our study's participants did not express more interest in SNP testing with behavior risk modifiers, suggesting that genomic testing results alone may not motivate health behavior change. A population-based study comparing relatives of colorectal cancer patients to controls found that $25 \%$ of participants with a hypothetical positive predictive genomic test result would exercise 'a lot more' while $30 \%$ claimed they would make significant dietary changes. There was no significant difference between relatives and controls [9]. However, intentions to change behavior based on a hypothetical test result do not necessarily reflect actual behavior modifications influenced by genomic testing. In another study, women at increased risk for hereditary breast cancer reported statistically less interest in SNP testing with behavior-risk modifiers, like daily vitamins or exercise and healthy diets, than testing for genetic factors that conferred risk for breast cancer independent of lifestyle factors [16]. Similarly, there was no significant difference in dietary fat intake, exercise and screening behaviors from baseline to follow-up in adults who received DTC genome-wide profiling [36]. Current evidence suggests that although genetic susceptibility information may function as a cue to action or catalyst for behavior modification, it may not be enough to motivate sustained health behavior change [37, 38]. Genomic test results that confer a modest increase in cancer risk may result in health behavior change among highly motivated individuals. But, most consumers of genomic testing likely need action-oriented health counseling or other evidence-based health education strategies to enact behavior change following personalized genomic testing [39]. It is not clear, however, if genomic testing adds value to evidence-based behavioral interventions. More research in this area is needed to establish the clinical utility of SNP and other genomic testing for common risk variants.

Age, marital status, intervention group, cancer worry, perceived cancer risk, and cancer fear achieved crude statistical significance in the bivariate analysis. Age, marital status, intervention group, and cancer-related fear were independently associated with interest in genomic testing in the overall study population. Cancer-related fear was associated with interest in SNP testing in the intervention group, but not in the control group. The intervention in this study was designed to raise cancer-related fear while also providing participants with efficacy tools to manage this fear. As such, some of the intervention participants in this study may have residual fear that influences protective motivation processes, and thus, the desire for SNP testing. Fear has been shown to motivate behavior, leading individuals to seek ways of removing or coping with the health threat or danger [40]. In one study, genetic counseling before and after testing for non-polyposis colorectal cancer relieved cancer fear in participants that tested either mutation positive or mutation negative after 1 year of follow-up [41]. In the future, it may be important to determine if and how cancer fear changes with receipt of SNP genomic test results that confer modest to moderate cancer risk over time and behavior modification scenarios. DOI: $10.1159 / 000356567$
Anderson et al. 
Prior research found cancer worry to be positively associated with interest in predictive SNP testing for modest changes in breast cancer risk [ 16,42$]$. However, in our study cancer-related fear was the only emotional factor independently associated with interest in testing, consistent with the Common Sense Model of Self Regulation. Cancer worry was associated with interest in SNP testing in the crude analysis, but did not remain significant in the multivariable models, perhaps because cancer worry was moderately correlated with fear (data not shown). We collapsed cancer worry into 3 categories - low, moderate and high - because of small numbers, preventing a more granulated analysis of cancer worry. Constructs included in the cognitive process (i.e. perceived risk, perceived severity and perceived susceptibility) were not independently associated with interest in testing, suggesting that emotions may be more important factors than cognitions in this context.

Age was the most powerful factor associated with interest in predictive SNP testing for the control group and the overall study population, with the youngest age group expressing the greatest interest. Interest in testing decreased with increasing age group. Research shows that younger age groups demonstrate higher health and genetic literacy and are more receptive to emerging technologies than older populations [32]. With higher health and genetic literacy, younger participants may more fully understand the genomic testing information provided in the 9-month follow-up questionnaire which may lead to greater interest in testing. A prior study found that participants who were confident in their ability to understand genomic information were more likely to express interest in SNP testing for multiple common health conditions [33]. Also, younger age groups may find genetic testing more advantageous, possibly because they have more years of life left to engage in health behavior change and benefit from knowing personal genomic cancer risk. For example, a survey on public interest in personal genomic testing found that younger age groups were more likely to support genomic testing to learn more about oneself [18]. Age was not associated with testing interest in the intervention group. But, the family risk information and behavior change counseling session with the intervention group may have mitigated differences in levels of genomic understanding across age groups, at least to some degree.

Unmarried individuals were significantly more interested in genomic testing than married participants in the total study population and in the control group. This was unexpected, as marital status was either not associated

Interest in Genomic Testing for Colorectal Cancer Risk with genomic testing interest in prior studies $[16,17]$ or married individuals were more interested in testing than unmarried participants $[43,44]$. Participants who responded to the 9-month survey were more likely to be married compared to nonrespondents, possibly introducing response bias. We may be underestimating interest in SNP testing for unmarried individuals. Members of an unmarried couple, separated, divorced, widowed, and never married participants were all considered 'unmarried' because of small numbers, precluding a more granular analysis. We were unable to determine if participants in these groups were more or less likely than married individuals to express interest in genomic testing. Sharing genetic risk information with family members, specifically children, is frequently cited as an advantage of genomic testing [17-19]. As such, parental status may moderate the relationship between marital status and interest in genomic testing. Unfortunately, we did not measure whether or not participants have children in our study, but future research should consider parental status as a possible factor associated with interest in genomic testing.

Those in the control arm of the Family CARE study, receiving only an educational brochure targeted at their familial risk group, expressed significantly more interest in predictive SNP testing for colorectal cancer risk than those randomized to the higher intensity TeleCARE intervention arm of the study. During the genetic counseling session, participants in the TeleCARE arm of the study received extensive information on how family history contributes to colorectal cancer risk, but did not necessarily learn about genomic testing. Families with known familial adenomatous polyposis or meeting Amsterdam criteria for Lynch syndrome were excluded from the study; as such, most participants did not receive any recommendation for genomic testing from the genetic counselors. Some participants did inquire about genetic testing for colorectal cancer on their own; however, most did not have a family history that warranted genetic testing for any high-risk cancer syndromes. Many TeleCARE participants likely felt they already understood their colorectal cancer risk based on the family history risk assessment they received as part of the Family CARE study and consequently, would not benefit from personal genomic testing. The control group, on the other hand, may have felt less informed about their familial and genetic risk of colorectal cancer and, therefore, be more interested in pursuing additional information about their personal genomic risk. Differences in the intervention content and dose between the 2 arms may explain why the 
control group expressed greater interest in SNP testing than the intervention group.

Participants most frequently cited print sources and physicians as their preferred methods for receiving genomic risk communications. During the Family CARE study, all participants received information on familial colorectal cancer risk via mailed educational brochures. TeleCARE participants also received tailored mailed print materials and one-on-one telephone education and counseling by cancer risk specialists. In this case, participants' familiarity with print materials to communicate cancer risk may contribute to the high proportion of respondents endorsing print/written sources. Printed materials allow individuals to read and digest information at their own pace, but lack interaction with a physician or genetic counselor. In similar studies, people also preferred that genetic risk information be delivered in person by a healthcare provider, such as a primary care physician or oncologist $[17,45]$. However, available evidence suggests that most primary care providers lack both time and expertise to effectively communicate genomic risk information with patients. A survey of primary care providers found that only $39 \%$ of physicians were aware of DTC genomic testing and $85 \%$ of physicians felt unprepared to discuss genomic testing with patients [46]. Early adopters of personalized genomics not only intended to share their testing results with a physician, but also expected their physician to tailor their healthcare needs based on personal genomic information [47]. There are currently no clinical guidelines or evidence to support health interventions following SNP testing (e.g. screening frequency, diet/lifestyle interventions) possibly making clinicians even more hesitant to interpret SNP results for patients. Without adequate time and sufficient knowledge, primary care physicians cannot meet such demands. It is important to note that these studies only evaluated intentions to consult primary care providers, not actions. But, a recent study reported that $27 \%$ of adults who received DTC genomic testing shared their results with a healthcare provider; those sharing their testing results with a provider were more health conscious and expressed fewer genomic-related privacy concerns [39]. Predictive SNP testing for common health-related conditions cannot be effectively translated into clinical practice without both patients and providers understanding the implications of genomic testing.

Limitations to this study deserve discussion. First, we did not include the cost of predictive SNP testing in the survey. Graves et al. [16] found that interest in SNP testing for modest changes in breast cancer risk increased as test-associated costs decreased. Although we did not measure cost, it is still interesting to note that three-quarters of participants expressed interest in predictive SNP testing. Second, participants were only provided with a brief overview of genetics, disease risk and SNPs. Such a short introduction may not be enough information for participants to determine whether or not they are interested in SNP testing, especially those with low levels of health/genetic literacy. Future studies should assess interest and testing uptake after accounting for previous genetic knowledge and genetic literacy and following a more detailed discussion of the issues surrounding predictive SNP testing, like controversial clinical validity and utility. We only assessed whether or not participants were interested in SNP testing, not reasons for interest or actual uptake of genomic testing. Third, we recognize that our findings may not be generalizable to the whole population. Not all Family CARE participants completed the 9-month follow-up questionnaire resulting in possible response bias. However, respondents only differed from nonrespondents in gender and marital status. Our study population was rather homogenous and limited to nonHispanic whites, thereby limiting the generalizability of our findings. All study participants were participating in an intervention trial and were nonadherent to colorectal cancer screening guidelines. We might see different levels of interest in a different study sample. Our results may not reflect the attitudes of the general population because those who participate in research studies are different from those in the general population. Finally, the small sample size in some strata may have limited our statistical power to detect small differences. Results should be interpreted in context of these limitations.

Our study is a first step toward understanding interest in genomic testing for modest increases in disease risk and preferences for receiving genomic risk communications. Interest in predictive SNP testing for colorectal cancer was high among relatives of colorectal cancer cases. Participants preferred to receive genomic risk communications from a variety of sources including print, healthcare providers - such as primary care physicians and the web. Primary care physicians, however, may not be prepared to interpret genomic testing results or provide genomic risk communications [48, 49]. As such, educational interventions are needed for both patients and primary care providers to facilitate effective and appropriate translation of predictive genomic testing into clinical practice, especially when considering the expansion of DTC genetic testing. The DTC genetic testing market is currently unregulated with little oversight to ensure DOI: $10.1159 / 000356567$
Anderson et al. 
quality testing or full disclosure of the limitations of these tests, including their predictive value. Public interest in SNP testing may change, if these limitations are explicitly stated. If clinic validity and utility is established for certain SNP tests, these tests could conceivably become a standard of care. Behavioral intervention research can help guide future policy and clinical practice guidelines, like developing provisions for genetic education and counseling. Future research should also address dissemination of genomic risk communications to underserved populations who are less likely to seek genetic testing or to understand genetic risk results. Although we studied predictive SNP testing for colorectal cancer risk, the present findings apply to other genomic tests for modest increases in disease risk.

\section{Acknowledgements}

This paper includes Family Colorectal Cancer Awareness and Risk Education (Family CARE) Project data obtained from the Kinney Research Group and is registered on the ClinicalTrials. gov website (NCT01274143). Family CARE was funded by the National Cancer Institute (1R01CA125194-03; Kinney, PI) and the Huntsman Cancer Foundation. Family CARE was also supported by the Shared Resources (P30 CA042014) at Huntsman
Cancer Institute; the Utah Cancer Registry, which is funded by Contract No. HHSN261201000026C from the National Cancer Institute's SEER Program with additional support from the Utah State Department of Health and the University of Utah; the California Department of Public Health as part of the statewide cancer reporting program mandated by California Health and Safety Code Section 103885, the National Cancer Institute's Surveillance, Epidemiology and End Results Program under contract N01PC-2010-00034C awarded to the Northern California Cancer Center, contract N01-PC-35139 awarded to the University of Southern California, and contract N01-PC-54404 awarded to the Public Health Institute, and the Centers for Disease Control and Prevention's National Program of Cancer Registries, under agreement U58CCU000807-05 awarded to the Public Health Institute; the Colorado Central Cancer Registry program in the Colorado Department of Public Health and Environment funded by the National Program of Cancer Registries of the Centers for Disease Control and Prevention; the Cancer Data Registry of Idaho supported in part by the National Program of Cancer Registries of the Centers for Disease Control and Prevention; the New Mexico Tumor Registry which is funded by National Cancer Institute Contract No. HHSN261201000033C; the Rocky Mountain Cancer Genetics Network (HHSN261200744000C); the Huntsman Cancer Registry; the University of Utah Department of Orthopaedics and the Center for Outcomes Research and Assessment; the Intermountain Healthcare Oncology Clinical Program and Intermountain Clinical Genetics Institute. This content is solely the responsibility of the authors and does not necessarily reflect the opinions or views of the funding and supporting agencies.

\section{References}

1 Jasperson KW, Tuohy TM, Neklason DW, Burt RW: Hereditary and familial colon cancer. Gastroenterology 2010;138:2044-2058.

2 National Comprehensive Cancer Network: Clinical practice guidelines in oncology: colorectal cancer screening. Version 22013. http://www.nccn.org.

-3 Evaluation of Genomic Applications in Practice and Prevention (EGAPP) Working Group: Recommendations from the EGAPP working group: genetic testing strategies in newly diagnosed individuals with colorectal cancer aimed at reducing morbidity and mortality from lynch syndrome in relatives. Genet Med 2009;11:35-41.

- 4 Ait Ouakrim D, Lockett T, Boussioutas A, Hopper JL, Jenkins MA: Screening participation for people at increased risk of colorectal cancer due to family history: a systematic review of meta-analysis. Fam Cancer 2013;12: 459-472.

5 Taylor DP, Burt RW, Williams MS, Haug PJ, Cannon-Albright LA: Population-based family history-specific risks for colorectal cancer: a constellation approach. Gastroenterology 2010;138:877-885.
6 American Cancer Society: American cancer society recommendations for colorectal cancer early detection. http://www.cancerorg/ cancer/colonandrectumcancer/moreinformation/colonandrectumcancerearlydetection/colorectal-cancer-early-detection-acsrecommendations.

7 Siegert S, Hampe J, Schafmayer C, von Schönfels W, Egberts JH, Försti A, Chen B, Lascorz J, Hemminki K, Franke A, Nothnagel M, Nöthlings U, Krawczak M: Genome-wide investigation of gene-environment interactions in colorectal cancer. Hum Genet 2013;132: 219-231.

-8 Fugger L, McVean G, Bell JI: Genomewide association studies and common disease - realizing clinical utility. N Engl J Med 2012;367: 2370-2371.

-9 Ramsey S, Blough D, McDermott C, Clarke L, Bennett R, Burke W, Newcomb P: Will knowledge of gene-based colorectal cancer disease risk influence quality of life and screening behavior? Findings from a population-based study. Public Health Genomics 2010;13:1-12.
10 Dvoskin R, Kaufman D: Tables of direct-toconsumer genetic testing companies and conditions tested - August 2011. Washington, DC, Genetics and Public Policy Center, 2011. http://www.dnapolicy.org/pub.reports.php (accessed August 24, 2013).

11 23andMe: 2013. https://www.23andme.com/ (accessed January, 5 2013).

12 Accu-metrics/Viaguard: http://www.accumetrics.com/cancer-scan.Php (accessed August 24, 2013).

13 Herper M, Langreth R: Will you get cancer? Forbes 2007;179:52-68.

14 Wright CF, Gregory-Jones S: Size of the direct-to-consumer genomic testing market. Genetics 2010;12:594.

15 Saukko P: State of play in direct-to-consumer genetic testing for lifestyle-related diseases: market, marketing content, user experiences and regulation. Proc Nutr Soc 2013;72:53-60.

16 Graves KD, Peshkin BN, Luta G, Tuong W, Schwartz MD: Interest in genetic testing for modest changes in breast cancer risk: implications for SNP testing. Public Health Genomics 2011;14:178-189. 
17 Leventhal KG, Tuong W, Peshkin BN, Salehizadeh Y, Fishman MB, Eggly S, FitzGerald $\mathrm{K}$, Schwartz MD, Graves KD: 'Is it really worth it to get tested?': primary care patients' impressions of predictive SNP testing for colon cancer. J Genet Couns 2013;22:138-151.

18 Cherkas LF, Harris JM, Levinson E, Spector TD, Prainsack B: A survey of UK public interest in internet-based personal genome testing. PloS One 2010;5:e13473.

19 McBride CM, Wade CH, Kaphingst KA: Consumers' views of direct-to-consumer genetic information. Ann Rev Genomics Hum Genet 2010;11:427-446.

20 Leventhal H, Brissette I, Leventhal EA: The common-sense model of self-regulation of health and illness; in Cameron LD, Leventhal $\mathrm{H}$ (eds): The Self-Regulation of Health and Illness Behaviour. London and New York, Routledge, 2003, pp 42-65.

21 Marteau TM, Weinman J: Self-regulation and the behavioural response to DNA risk information: a theoretical analysis and framework for future research. Soc Sci Med 2006;62: 1360-1368.

22 Pengchit W, Walters ST, Simmons RG, Kohlmann W, Burt RW, Schwartz MD, Kinney AY: Motivation-based intervention to promote colonoscopy screening: an integration of a fear management model and motivational interviewing. J Health Psychol 2011;16: 1187-1197.

-23 Simmons RG, Lee YC, Stroup AM, Edwards SL, Rogers A, Johnson C, Wiggins CL, Hill DA, Cress RD, Lowery J, Walters ST, Jasperson K, Higginbotham JC, Williams MS, Burt RW, Schwartz MD, Kinney AY: Examining the challenges of family recruitment to behavioral intervention trials: factors associated with participation and enrollment in a multistate colonoscopy intervention trial. Trials 2013; 14:116.

-24 O’Neill SC, Bowling JM, Brewer NT, Lipkus IM, Skinner CS, Strigo TS, Rimer BK: Intentions to maintain adherence to mammography. J Womens Health (Larchmt) 2008;17: 1133-1141.

25 Witte K, Meyer G, Martell D: Effective health risk messages: a step-by-step guide. California, Sage Publications, Inc., 2001.

26 Costa PT, McCrae RR: Normal personality assessment in clinical practice: the neo personality inventory. Psychol Assess 1992;4:5-13.
27 McCaul KD, Goetz PW: National Cancer Institute: Worry. http://dccps.nci.nih.gov/BRP/ constructs/worry/index.html (accessed December 5, 2013).

28 Hay JL, Primavera L, Gurmankin Levy A, et al: Development and validation of a scale assessing novel cancer-related risk perceptions. Abstracts 27th Annu Meet Society for Behavioral Medicine. San Francisco, March, 2006.

29 Scheier MF, Carver CS, Bridges MW: Distinguishing optimism from neuroticism (and trait anxiety, self-mastery, and self-esteem): a reevaluation of the Life Orientation Test. J Pers Soc Psychol 1994;67:1063-1078.

30 Rural Health Research Center University of Washington: Rural-urban commuting areas (RUCAS). Seattle, University of Washington, 2007.

31 US Department of Health and Human Services: Physical activity guidelines for Americans, 2008. http://www.Health.Gov/paguidelines/factsheetprof.Aspx (accessed January 10, 2013).

32 Centers for Disease Control and Prevention: About BMI for adults. http://www.Cdc.Gov/ healthyweight/assessing/bmi/adult_bmi/index.Html (accessed January 10, 2013).

33 Burton AM, Peterson SK, Marani SK, Vernon SW, Amos CI, Frazier ML, Lynch PM, Gritz ER: Health and lifestyle behaviors among persons at risk of Lynch syndrome. Cancer Causes Control 2010;21:513-521.

- 34 Emmons KM, McBride CM, Puleo E, Pollak KI, Marcus BH, Napolitano M, Clipp E, Onken J, Farraye FA, Fletcher R: Prevalence and predictors of multiple behavioral risk factors for colon cancer. Prev Med 2005;40:527-534.

- 35 McBride CM, Bryan AD, Bray MS, Swan GE, Green ED: Health behavior change: can genomics improve behavioral adherence? Am J Public Health 2012;102:401-405.

36 Bloss CS, Schork NJ, Topol EJ: Effect of direct-to-consumer genomewide profiling to assess disease risk. N Engl J Med 2011;364: 524-534.

37 Marteau TM, French DP, Griffin SJ, Prevost AT, Sutton S, Watkinson C, Attwood S, Hollands GJ: Effects of communicating DNAbased disease risk estimates on risk-reducing behaviours. Cochrane Database Syst Rev 2010;CD007275.

38 Schully SD, Benedicto CB, Khoury MJ: How can we stimulate translational research in cancer genomics beyond bench to bedside? Genet Med 2012;14:169-170.

-39 Bloss CS, Madlensky L, Schork NJ, Topol EJ: Genomic information as a behavioral health intervention: can it work? Per Med 2011;8: 659-667.
40 So JY: A further extension of the extended parallel process model (E-EPPM): implications of cognitive appraisal theory of emotion and dispositional coping style. Health Commun 2013;28:72-83.

41 Aktan-Collan K, Haukkala A, Mecklin JP, Uutela A, Kääriäinen H: Psychological consequences of predictive genetic testing for hereditary non-polyposis colorectal cancer (HNPCC): a prospective follow-up study. Int J Cancer 2001;93:608-611.

42 Cameron LD, Reeve J: Risk perceptions, worry, and attitudes about genetic testing for breast cancer susceptibility. Psychol Health 2006;21:211-230.

43 Bruno M, Tommasi S, Stea B, Quaranta M, Schittulli F, Mastropasqua A, Distante A, Di Paola L, Paradiso A: Awareness of breast cancer genetics and interest in predictive genetic testing: a survey of a southern Italian population. Ann Oncol 2004;15(suppl 1):I48-I54.

44 Weinrich S, Royal C, Pettaway CA, Dunston G, Faison-Smith L, Priest JH, RobersonSmith P, Frost J, Jenkins J, Brooks KA, Powell I: Interest in genetic prostate cancer susceptibility testing among african American men. Cancer Nurs 2002;25:28-34

45 McGuire AL, Diaz CM, Wang T, Hilsenbeck SG: Social networkers' attitudes toward direct-to-consumer personal genome testing. Am J Bioeth 2009;9:3-10.

46 Powell KP, Christianson CA, Cogswell WA, Dave G, Verma A, Eubanks S, Henrich VC: Education needs of primary care physicians regarding direct-to-consumer genetic testing. J Genet Couns 2012;21:469-478.

47 Gollust SE, Gordon ES, Zayac C, Griffin G, Christman MF, Pyeritz RE, Wawak L, Bernhardt BA: Motivations and perceptions of early adopters of personalized genomics: perspectives from research participants. Public Health Genomics 2012;15:22-30.

48 Birmingham WC, Agarwal N, Kohlmann W, Aspinwall LG, Wang M, Bishoff J, Dechet C, Kinney AY: Patient and provider attitudes toward genomic testing for prostate cancer susceptibility: a mixed method study. BMC Health Serv Res 2013;13:279.

49 Haga SB, Carrig MM, O’Daniel JM, Orlando LA, Killeya-Jones LA, Ginsburg GS, Cho A: Genomic risk profiling: attitudes and use in personal and clinical care of primary care physicians who offer risk profiling. J Gen Intern Med 2011;26:834-840. 